\title{
ESQUEMA PARA INTERPRETAÇÃO DE ESPECTROS DE SUBSTÂNCIAS ORGÂNICAS NA REGIÃO DO INFRAVERMELHO
}

\author{
Wilson Araújo Lopes* e Miguel Fascio \\ Instituto de Química, Universidade Federal da Bahia, Campus Universitário de Ondina, 40170-290 Salvador - BA
}

Recebido em 25/7/03; aceito em 27/11/03; publicado na web em 27/05/04

\begin{abstract}
FLOW CHART FOR INFRARED SPECTRA INTERPRETATION OF ORGANIC COMPOUNDS. This article decribes a simple and systematic method to interpret an infrared spectrum using a flow chart to elucidate the structure of a simple organic compound. It is aimed at undergraduate courses of organic chemistry to make beginners proficient. The proposed flow chart for infrared spectrum interpretation and characterization of organic compounds is suitable for theoretical and experimental courses.
\end{abstract}

Keywords: infrared spectroscopy; infrared spectra interpretation; infrared spectra of organic compounds.

\section{INTRODUÇÃO}

A espectroscopia na região do infravermelho (IV) é uma técnica de inestimável importância na análise orgânica qualitativa, sendo amplamente utilizada nas áreas de química de produtos naturais, síntese e transformações orgânicas. O infravermelho e demais métodos espectroscópicos modernos como a ressonância magnética nuclear (RMN), espectroscopia na região do ultravioleta-visível (UV-VIS) e espectrometria de massas (EM) constituem hoje os principais recursos para a identificação e elucidação estrutural de substâncias orgânicas. São, também, de alta relevância na determinação da pureza e quantificação de substâncias orgânicas, bem como no controle e acompanhamento de reações e processos de separação. O uso dos referidos métodos físicos de análise traz uma série de vantagens, destacando-se a redução no tempo de análise, diminuição substancial nas quantidades de amostra, ampliação da capacidade de identificar ou caracterizar estruturas complexas, não destruição da amostra (exceto EM) e a possibilidade de acoplamento com métodos modernos de separação, como a cromatografia gasosa de alta resolução (CGAR) e cromatografia líquida de alta eficiência (CLAE). A espectroscopia na região do infravermelho tem sido, também, amplamente utilizada em linhas de produção, no controle de processos industriais.

A interpretação de espectros de infravermelho de substâncias orgânicas é uma tarefa que, devido ao grande número de informações que devem ou precisam ser manipuladas, geralmente apresenta dificuldades para os alunos dos cursos básicos de graduação. Assim, o estudante iniciante necessita de um meio para, de modo sistemático, interpretar um espectro na região do infravermelho e propor uma possível estrutura molecular. Os livros textos geralmente apresentam tabelas de correlação entre as absorções de estiramento e deformação, em número de onda (4000 - $\left.400 \mathrm{~cm}^{-1}\right)$ e/ou comprimento de onda $(2,5-25 \mu \mathrm{m})$, e os respectivos grupos funcionais ou ligações químicas correspondentes ${ }^{1-4}$. Não há, contudo, uma preocupação com a sistematização de um caminho que possibilite ao estudante analisar um espectro infravermelho, obter informações sobre as principais ligações e grupos funcionais de uma determinada substância orgânica e, finalmente, propor uma possível estrutura. Com a finalidade de orientar a análise e interpretação de espectros de infravermelho já foram publicados alguns artigos na literatura ${ }^{5-7}$ e o livro de Doyle e Mungall ${ }^{8}$ apresenta um esquema resumido para in-

*e-mail: willopes@ufba.br terpretação que, porém, é insuficiente para a identificação da maioria das substâncias orgânicas mais simples.

O presente trabalho representa uma contribuição com o objetivo de facilitar a tarefa de análise e interpretação de espectros de infravermelho, estabelecendo um caminho objetivo e prático que permite a identificação dos principais grupamentos funcionais e a proposição de uma possível estrutura (ou estruturas) para as substâncias orgânicas mais simples. Havendo necessidade, tabelas de correlação ${ }^{1-4,9}$ deverão ser consultadas para a completa interpretação dos espectros. A comparação da região de impressão digital (1400 a $900 \mathrm{~cm}^{-1}$ ) com o espectro de uma amostra padrão é de fundamental importância para confirmar a identidade da substância analisada.

É importante ressaltar que o esquema da Figura 1 vem sendo utilizado com pleno êxito nas disciplinas de Química Orgânica oferecidas aos alunos dos cursos de Química, Engenharia Química e Farmácia da Universidade Federal da Bahia, há mais de 10 anos. Este esquema é uma segunda versão, revisada e ampliada com a participação de alunos e a contribuição dos professores das disciplinas de Análise Orgânica, Química Orgânica Fundamental e Química Orgânica Básica Experimental.

\section{CALCULANDO O ÍNDICE DE DEFICIÊNCIA DE HIDROGÊNIO}

A determinação da fórmula estrutural de uma substância orgânica requer um conjunto de informações que envolvem propriedades químicas e físicas. O conhecimento da fórmula molecular representa uma importante contribuição pois permite calcular o Índice de Deficiência de Hidrogênio (IDH), que indica a ausência ou presença de ligações duplas, triplas ou anéis na estrutura molecular e, muitas vezes, tem sido indevidamente denominado de índice de insaturação o que, de fato, não corresponde à realidade. Os alcenos, devido à presença de uma ligação dupla carbono-carbono, e os ciclo-alcanos, por conterem um anel, têm dois hidrogênios a menos que os hidrocarbonetos acíclicos saturados de fórmula $\mathrm{C}_{\mathrm{n}} \mathrm{H}_{2 \mathrm{n}+2}$. Por exemplo, o eteno $\left(\mathrm{H}_{2} \mathrm{C}=\mathrm{CH}_{2}\right)$ tem fórmula molecular $\mathrm{C}_{2} \mathrm{H}_{4}$ e o etano $\left(\mathrm{H}_{3} \mathrm{C}\right.$ $\mathrm{CH}_{3}$ ) tem fórmula $\mathrm{C}_{2} \mathrm{H}_{6}$.

O cálculo do IDH pode se feito por mais de um método, destacando-se:

a) pela aplicação da expressão' ${ }^{1}$

$\mathrm{IDH}=(\mathrm{C}-\mathrm{M} / 2)+\mathrm{T} / 2+1$

$\mathrm{C}=$ número de átomos de carbono; $\mathrm{M}=$ número de átomos monovalentes e $\mathrm{T}=$ número de átomos trivalentes. 
b) Segundo as regras referidas por Klemm ${ }^{10,11}$, com base na comparação entre as fórmulas da substância desconhecida e o correspondente hidrocarboneto saturado (alcano):

1. substituir todos os átomos monovalentes (halogênios) por hidrogênio;

2. desconsiderar os átomos bivalentes (oxigênio e enxofre);

3. excluir os átomos trivalentes junto com um hidrogênio (nitrogênio como $\mathrm{NH}$ e fósforo como $\mathrm{PH}$ ) e

4. comparar com a fórmula geral dos hidrocarbonetos saturados $\left(\mathrm{C}_{\mathrm{n}} \mathrm{H}_{2 \mathrm{n}+2}\right)$.

\section{INTERPRETANDO UM ESPECTRO DE INFRAVERMELHO}

a) Seguindo o esquema da Figura 1, observar inicialmente a presença ou ausência de absorção devida ao grupamento carbonila. Se o espectro da substância apresentar absorção entre 1820 a $1630 \mathrm{~cm}^{-1}$ $(\mathrm{v}=\mathrm{O})$, seguir a seta à direita e identificar o grupo funcional res- ponsável pela absorção (ácido carboxílico, amida, aldeído, anidrido, éster, haleto de acila, cetona, aril-cetona). Seguir então para o bloco esquerdo do esquema e verificar a presença ou ausência de outras funções orgânicas. Se não apresentar absorção entre 1820 a $1630 \mathrm{~cm}^{-1}$, seguir a seta à esquerda e identificar as ligações ou grupos funcionais presentes ou ausentes na estrutura molecular. Havendo uma ligação dupla ou anel aromático, caracterizar o padrão de substituição nos quadros correspondentes: olefinas ou benzeno e derivados. Finalmente, verificar a presença de grupos $\mathrm{CH}_{2}$ e $\mathrm{CH}_{3}$ no quadro referente a alcanos.

b) Com base nas informações obtidas do espectro de infravermelho, fórmula molecular e índice de deficiência de hidrogênio identificar as principais ligações ou grupamentos, assinalar a função (ou funções) orgânica e propor uma possível estrutura (ou estruturas) para a substância que está sendo analisada.

As Figuras 2 a 5 mostram os espectros de infravermelho de quatro substâncias orgânicas (A-D) que, como exemplo, são analisadas de acordo com o esquema apresentado. As amostras foram purificadas

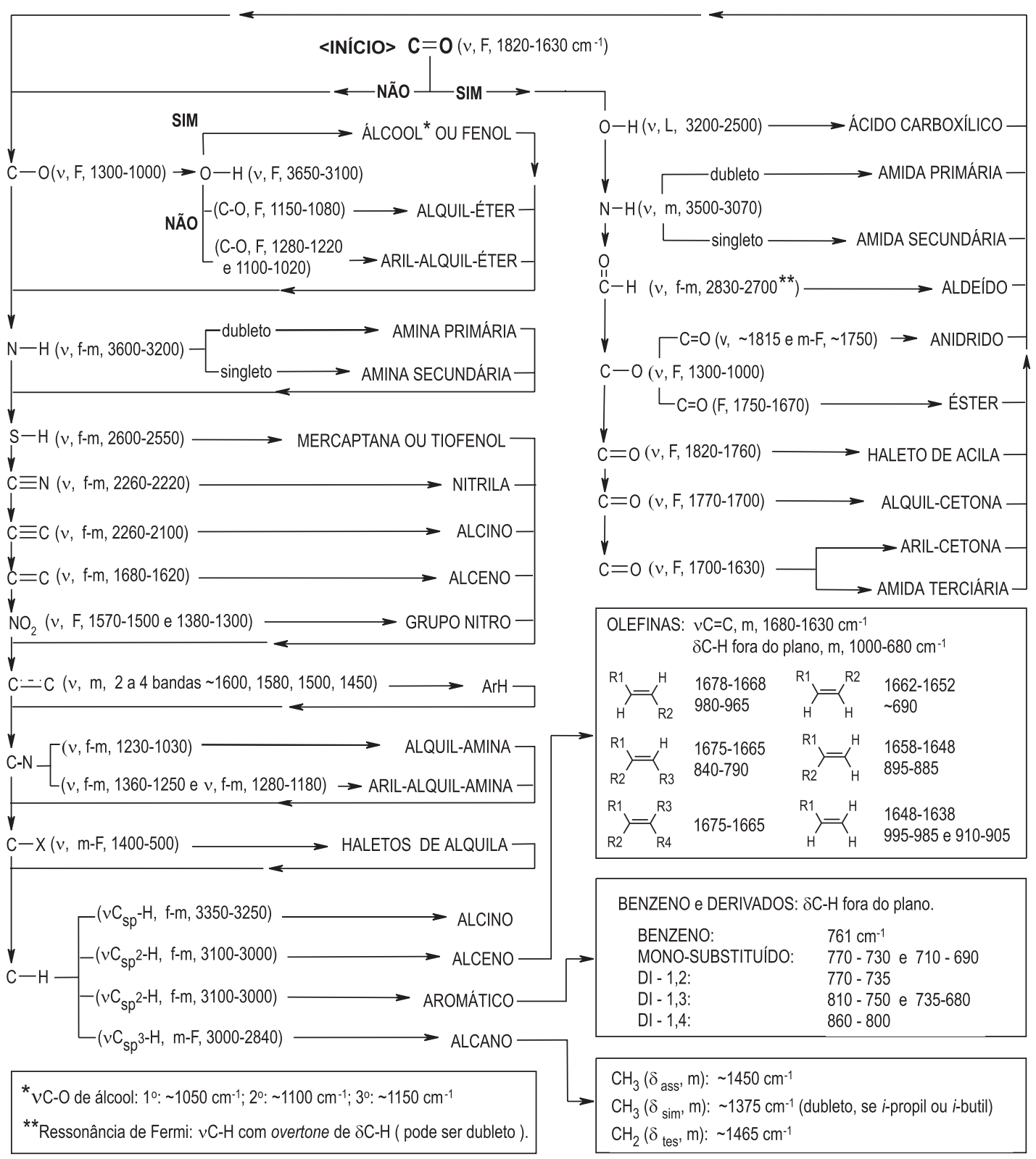

$v=$ estiramento; $\delta$ = deformação; ass = assimétrica; $\operatorname{sim}=$ simétriaca; tes = tesoura; $F=$ forte; $m=$ média; $f=$ fraca; $L=$ larga; $v=$ variável.

Figura 1. Esquema para interpretação de espectros de substâncias orgânicas na região do infravermelho 


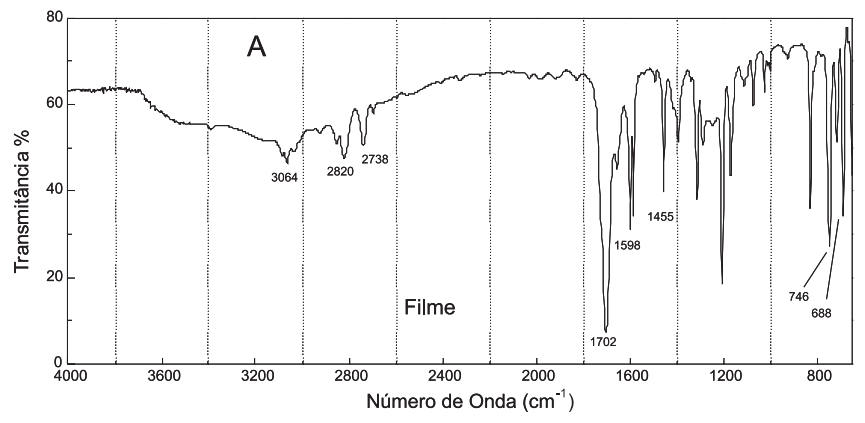

Figura 2. Espectro de infravermelho da substância A

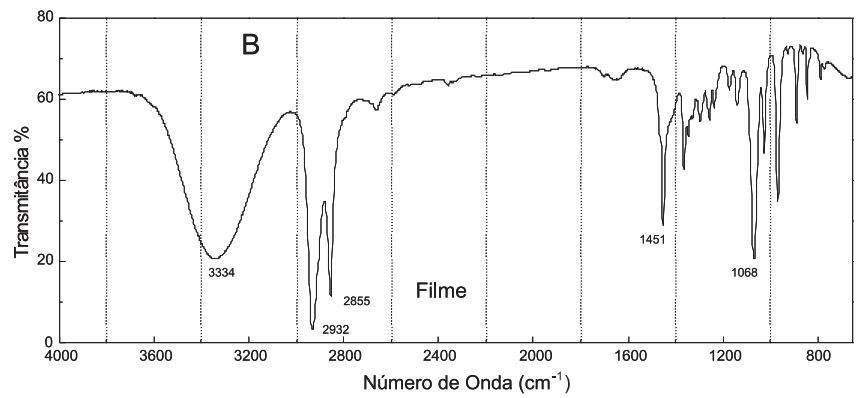

Figura 3. Espectro de infravermelho da substância $B$

Exemplos:

\begin{tabular}{lcccc}
\hline $\begin{array}{l}\text { Substância/ } \\
\text { estrutura }\end{array}$ & $\begin{array}{c}\text { Fórmula } \\
\text { molecular }\end{array}$ & $\begin{array}{c}\text { Fórmula } \\
\text { ajustada }\end{array}$ & $\begin{array}{c}\text { Fórmula } \\
\text { do Alcano }\end{array}$ & $\begin{array}{c}\text { IDH } \\
(2-1) / 2\end{array}$
\end{tabular}

(1) (2)

1.

$\checkmark \begin{array}{llll}\mathrm{C}_{4} \mathrm{H}_{8} & \mathrm{C}_{4} \mathrm{H}_{8} & \mathrm{C}_{4} \mathrm{H}_{10} & (10-8) / 2=1\end{array}$

2.<smiles>C1=CCCCC1</smiles>

$$
\mathrm{C}_{6} \mathrm{H}_{12} \quad \mathrm{C}_{6} \mathrm{H}_{12} \quad \mathrm{C}_{6} \mathrm{H}_{14} \quad(14-12) / 2=1
$$

3.<smiles>Clc1ccccc1</smiles>

$$
\mathrm{C}_{6} \mathrm{H}_{5} \mathrm{Cl}
$$

$\mathrm{C}_{6} \mathrm{H}_{6}$

$\mathrm{C}_{6} \mathrm{H}_{14}$

$(14-6) / 2=4$

4.<smiles>Cc1ccccc1</smiles>

$$
\mathrm{C}_{7} \mathrm{H}_{8}
$$

$\mathrm{C}_{7} \mathrm{H}_{8}$

$\mathrm{C}_{7} \mathrm{H}_{16}$

$(16-8) / 2=4$

5.<smiles>CCCC(=O)c1ccccc1</smiles>

$$
\mathrm{C}_{8} \mathrm{H}_{8} \mathrm{O}
$$

$\mathrm{C}_{8} \mathrm{H}_{8}$

$\mathrm{C}_{8} \mathrm{H}_{18}$

$(18-8) / 2=5$

6.

$$
\mathrm{C}_{5} \mathrm{H}_{11} \mathrm{~N}
$$

$\mathrm{C}_{5} \mathrm{H}_{10}$

$\mathrm{C}_{5} \mathrm{H}_{12}$

$(12-10) / 2=1$

por destilação (líquidos) ou cristalização (sólidos) e os espectros foram obtidos em Espectrômetro de Infravermelho com Transformada de Fourier (IV-TF), da marca Bomem, modelo ABB.

\section{Substância A $\left(\mathrm{C}_{7} \mathrm{H}_{6} \mathrm{O}\right)$ :}

Cálculo do IDH: $\quad(7-6 / 2)+1=5$ ou $\mathrm{C}_{7} \mathrm{H}_{16}-\mathrm{C}_{7} \mathrm{H}_{6}=\mathrm{H} 10 / 2=5$ Análise funcional: $1702 \mathrm{~cm}^{-1}, \mathrm{vC}=\mathrm{O}$;

2820 e $2738 \mathrm{~cm}^{-1}, v \mathrm{C}(=\mathrm{O})-\mathrm{H}$ (dubleto de Fermi); 1598 e $1455 \mathrm{~cm}^{-1}, \mathrm{v}=\mathrm{C}(\mathrm{ArH})$;

$3064 \mathrm{~cm}^{-1}, v \mathrm{Csp}^{2}-\mathrm{H} \mathrm{e}$

746 e $688 \mathrm{~cm}^{-1}, \delta \mathrm{C}-\mathrm{H}(\mathrm{ArH})$, monosubstituído.

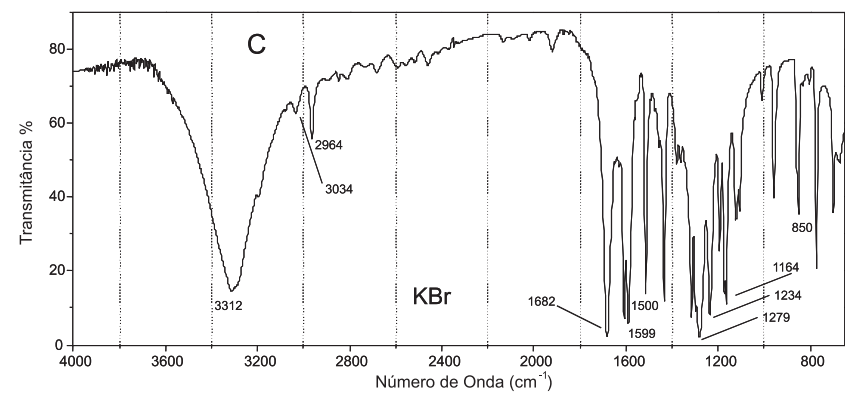

Figura 4. Espectro de infravermelho da substância C

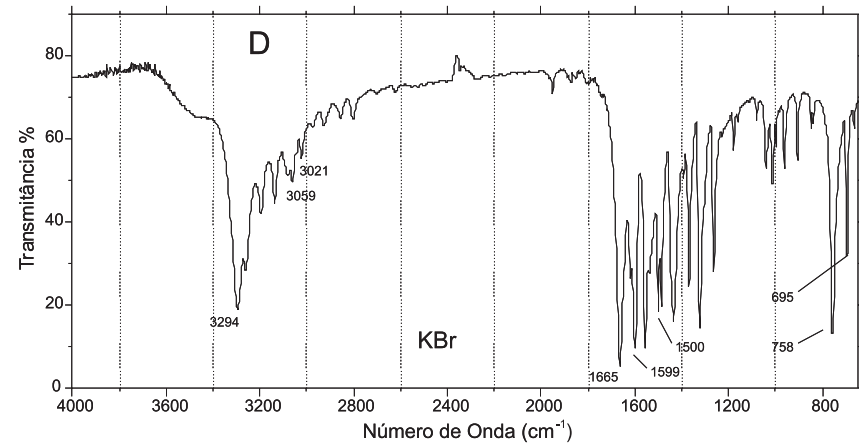

Figura 5. Espectro de infravermelho da substância D

Identificação: O IDH igual a 5 é compatível com a presença de um anel aromático. A presença de absorção a $1702 \mathrm{~cm}^{-1}(\mathrm{vC}=\mathrm{O})$ juntamente com o dubleto de Fermi (2820 e $\left.2738 \mathrm{~cm}^{-1}\right)$ é indicativo de função aldeído. As absorções a 1598 e $1455 \mathrm{~cm}^{-1},(v \mathrm{C}=\mathrm{C}, \mathrm{ArH})$ e $3064 \mathrm{~cm}^{-1},\left(\nu \mathrm{Csp}^{2}-\mathrm{H}\right)$ confirmam a presença de estrutura aromática. As absorções a 746 e $688 \mathrm{~cm}^{-1}(\delta \mathrm{C}-\mathrm{H}, \mathrm{ArH})$ indicam o padrão monossubstituído, concluindo-se que A corresponde ao benzaldeído. Estrutura:<smiles>O=Cc1ccccc1</smiles>

\section{Substância B $\left(\mathrm{C}_{6} \mathrm{H}_{12} \mathrm{O}\right)$ :}

Cálculo do IDH: $\quad(6-12 / 2)+1=1$ ou $\mathrm{C}_{6} \mathrm{H}_{14}-\mathrm{C}_{6} \mathrm{H}_{12}=\mathrm{H} 2 / 2=1$ Análise funcional: ausência de $v \mathrm{C}=\mathrm{O}$ entre 1820 e $1630 \mathrm{~cm}^{-1}$; $1068 \mathrm{~cm}^{-1}$, vC-O; ausência de $v \mathrm{C}=\mathrm{C}$ entre 1680 e $1620 \mathrm{~cm}^{-1} \mathrm{e}$ ausência de $\delta \mathrm{C}-\mathrm{H}$ em $\sim 1380 \mathrm{~cm}^{-1}$ (metila).

Identificação: O IDH igual a 1 é indicativo da presença de ligação dupla ou estrutura cíclica. A ausência de absorção entre 1820 e $1630 \mathrm{~cm}^{-1}$ exclui todas as funções carboniladas. As absorções em $1068 \mathrm{~cm}^{-1}$ (vC-O) e $3334 \mathrm{~cm}^{-1}$ (vO-H) são compatíveis com a função álcool. As absorções entre 3000 e $2800 \mathrm{~cm}^{-1}$ (vC-H) são compatíveis com a presença de carbono com hibridização $\mathrm{sp}^{3}$. A ausência de absorções entre 3100 e $3000 \mathrm{~cm}^{-1}\left(\mathrm{Csp}^{2}-\mathrm{H}\right)$ e entre 1680 e $1620 \mathrm{~cm}^{-1}$ $(\vee \mathrm{C}=\mathrm{C})$ elimina a possibilidade de ser uma olefina. A ausência de absorção em $\sim 1380 \mathrm{~cm}^{-1}$ ( $\left.\delta \mathrm{C}-\mathrm{H}\right)$ indica que a substância não possui grupo metila. $\mathrm{O}$ espectro de $\mathrm{B}$, portanto, poderá corresponder ao ciclo-hexanol (I) ou ciclopentanometanol (II). Pela comparação com o espectro de infravermelho de uma amostra autêntica é possível concluir que se trata do ciclo-hexanol (I).

Estruturas: (I )

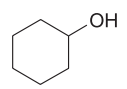

(II)

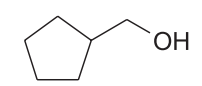


Substância C $\left(\mathrm{C}_{8} \mathrm{H}_{8} \mathrm{O}_{3}\right)$ :

Cálculo do IDH: $\quad(8-8 / 2)+1=5$ ou $\mathrm{C}_{8} \mathrm{H}_{18}-\mathrm{C}_{8} \mathrm{H}_{8}=\mathrm{H} 10 / 2=5$

Análise funcional: $1682 \mathrm{~cm}^{-1}, \mathrm{vC}=\mathrm{O}$;

1300 - $1100 \mathrm{~cm}^{-1}, v \mathrm{C}-\mathrm{O}$;

$3312 \mathrm{~cm}^{-1}, \mathrm{vO}-\mathrm{H}$;

1599 e $1500 \mathrm{~cm}^{-1}, \mathrm{vC}=\mathrm{C}(\mathrm{ArH})$;

$3034 \mathrm{~cm}^{-1}, v \mathrm{Csp}^{2}-\mathrm{H}$;

$2964 \mathrm{~cm}^{-1}, v \mathrm{Csp}^{3}-\mathrm{H} \mathrm{e}$

$850 \mathrm{~cm}^{-1}, \delta \mathrm{C}-\mathrm{H}(\mathrm{ArH})$, 1,4-di-substituído.

Identificação: $\mathrm{O}$ IDH igual a 5 é compatível com a presença de um anel aromático. A presença de uma banda de absorção em $1682 \mathrm{~cm}^{-1}$ $(v \mathrm{C}=\mathrm{O})$ associada com absorção entre 1300 e $1000 \mathrm{~cm}^{-1}(\mathrm{vC}-\mathrm{O})$ é indicativa da função éster. As absorções entre 1300 e $1100 \mathrm{~cm}^{-1}(\mathrm{vC}$ O) e a presença de banda de absorção a $3312 \mathrm{~cm}^{-1}$ (vO-H) permitem assinalar que a substância tem função mista - éster e fenol. A absorção em $2964 \mathrm{~cm}^{-1}$ é característica de $v \mathrm{Csp}^{3}-\mathrm{H}$ e as absorções em 1599 e $1500 \mathrm{~cm}^{-1}(\mathrm{vC}=\mathrm{C}, \mathrm{ArH})$ e em $3034 \mathrm{~cm}^{-1}\left(\mathrm{vCsp}^{2}-\mathrm{H}\right)$ confirmam a presença de estrutura aromática. A absorção em $850 \mathrm{~cm}^{-1}$ ( $\delta \mathrm{C}-\mathrm{H}, \mathrm{ArH})$ indica o padrão 1,4-dissubstituído, concluindo-se que C poderá corresponder ao 4-hidróxi-benzoato de metila (I) ou ao monoacetato de hidroquinona (II). Por meio do ponto de fusão (pf) e pela comparação com o espectro de infravermelho de uma amostra autêntica é possível concluir que se trata do 4-hidróxi-benzoato de metila (I).
Estruturas: (I )<smiles>COC(=O)c1ccc(O)cc1</smiles>

pf: $128^{\circ} \mathrm{C}$;
(II)

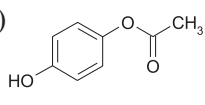

pf: $62{ }^{\circ} \mathrm{C}$

\section{Substância D $\left(\mathrm{C}_{8} \mathrm{H}_{9} \mathrm{NO}\right)$ :}

Cálculo do IDH: $(8-9 / 2)+1 / 2+1=5$ ou $\mathrm{C}_{8} \mathrm{H}_{18}-\mathrm{C}_{8} \mathrm{H}_{8}=\mathrm{H} 10 / 2=5$ Análise funcional: $1665 \mathrm{~cm}^{-1}, \mathrm{vC}=\mathrm{O}$;

$$
3294 \mathrm{~cm}^{-1}, v \mathrm{~N}-\mathrm{H}
$$

1599 e $1500 \mathrm{~cm}^{-1}, v \mathrm{C}=\mathrm{C}(\mathrm{ArH})$

3059 e $3021 \mathrm{~cm}^{-1}, \mathrm{vCsp}^{2}-\mathrm{H}$ e

758 e $695 \mathrm{~cm}^{-1}, \delta \mathrm{C}-\mathrm{H}(\mathrm{ArH})$, monossubstituído.

Identificação: O IDH igual a 5 é compatível com a presença de um anel aromático. A banda de absorção em $1665 \mathrm{~cm}^{-1}(\mathrm{vC}=\mathrm{O})$ associada à absorção em $3294 \mathrm{~cm}^{-1}(\mathrm{vN}-\mathrm{H})$ é indicativa da função amida. As absorções em 1599 e $1500 \mathrm{~cm}^{-1}(\mathrm{vC}=\mathrm{C}, \mathrm{ArH})$ e em 3059 e $3021 \mathrm{~cm}^{-1}$ $\left(v \mathrm{Csp}^{2}-\mathrm{H}\right)$ confirmam a presença de estrutura aromática. As absorções em 758 e $695 \mathrm{~cm}^{-1}$ ( $\left.\delta \mathrm{C}-\mathrm{H}, \mathrm{ArH}\right)$ indicam o padrão monossubstituído, concluindo-se que $\mathrm{D}$ poderá corresponder à $\mathrm{N}$-fenilacetamida (I) ou à N-metilbenzamida (II). Por meio do ponto de fusão (pf) e pela comparação com o espectro de infravermelho de uma amostra autêntica é possível concluir que se trata da $\mathrm{N}$-fenilacetamida (I).
Estruturas: (I )<smiles>CC(=O)Nc1ccccc1</smiles>

pf: $114^{\circ} \mathrm{C}$;
(II)<smiles>CNC(=O)c1ccccc1</smiles>

pf: $78^{\circ} \mathrm{C}$

\section{CONCLUSÕES}

O esquema proposto para interpretação de espectros na região do infravermelho permite, de modo simples e prático, a identificação dos principais grupos funcionais e a proposição de estruturas de substâncias orgânicas constituindo-se, assim, em recurso de grande utilidade no ensino de disciplinas tanto teóricas quanto experimentais.

\section{AGRADECIMENTOS}

Os autores agradecem aos alunos que participaram e contribuíram para o aperfeiçoamento do esquema apresentado, ao Prof. S. do D. Cunha (IQ-UFBA) pela leitura do texto e valiosas sugestões e à Profa. Z. N. da Rocha (IQ-UFBA) pelas facilidades oferecidas para utilização do aparelho de infravermelho.

\section{REFERÊNCIAS}

1. Silverstein, R. M.; Bassler, G. C.; Morrill, T. C.; Spectrometric Identification of Organic Compounds, $5^{\text {th. }}$ ed., John Wiley \& Sons: New York, 1991.

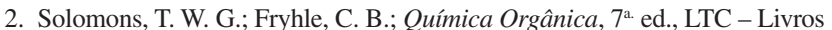
Técnicos e Científicos Editora S.A: Rio de Janeiro, 2001.

3. Nakanishi, K.; Solomon, P. H.; Infrared Absortion Spectroscopy, $2^{\text {nd. }}$ ed., Holden-Day Inc: Oakland, 1977.

4. Coates, J.; Em Encyclopedia Analytical Chemistry; Meyers, R. A., ed.; John Wiley \& Sons: Chichester, 2000, p. 10815 - 10837; http:// www.spectroscopynow.com:1800/Spy/pdfs/eac10815.pdf, acessada em Julho 2003.

5. Ingham, A. M.; Henson, R. C.; J. Chem. Educ. 1984, 61, 704.

6. Moyé, A. L.; Cochran Jr., T. A.; J. Chem. Educ. 1972, 49, 129.

7. Hartman, K.; J. Chem. Educ. 1976, 53, 111.

8. Doyle, M.P.; Mungall, W.S.; Experimental Organic Chemistry, John Wiley \& Sons: New York, 1980.

9. http://www.cem.msu.edu/\%7Ereusch/VirtualText/Spectrpy/InfraRed/ infrared.htm\#ir1, acessada em Julho 2003.

10. Kleman, L. H.; J. Chem. Educ. 1995, 72, 425.

11. http://chipo.chem.uic.edu/web1/ocol/SB/du.htm, acessada em Julho 2003. 